J. DIFFERENTIAL GEOMETRY

50 (1998) $123-127$

\title{
THE FINITENESS OF THE MAPPING CLASS GROUP FOR ATOROIDAL 3-MANIFOLDS WITH GENUINE LAMINATIONS
}

\author{
DAVID GABAI \& WILLIAM H. KAZEZ
}

Essential laminations were introduced in [7] in order to generalize the notion of taut foliation and incompressible surface. The papers $[7],[4],[5],[1]$, [3] have partially realized the hope that manifolds with essential laminations share many of the properties of Haken manifolds. The main result of this paper establishes the finiteness of the mapping class group, for the class of atoroidal 3-manifolds containing genuine laminations. A genuine lamination $\lambda$ is an essential lamination such that some closed complementary region is not an I-bundle. These are laminations which are genuinely not foliations, that is, they are not obtained by splitting open along leaves (if $\lambda$ has no isolated leaves). See [2] for an extensive list of constructions of essential laminations in 3-manifolds, many of which also yield genuine laminations. See [7] for background information on essential laminations.

Notation. $\stackrel{\circ}{E}$ denotes the interior of $E$, and $|E|$ denotes the number of components of $E .1_{X}$ is the identity map of $X$. Homeo $(M)$ is the group of homeomorphisms of $M$, and Homeo $_{0}(M)$ is the group of homeomorphisms of $M$ which are isotopic to $1_{M}$. The mapping class group, $\operatorname{Homeo}(M) / \operatorname{Homeo}_{0}(M)$, is denoted $\mathcal{M}(M)$. If $\alpha \subset M$ is a simple closed curve, then $I_{\alpha}$ is the subset of $\mathcal{M}(M)$ defined by $I_{\alpha}=$ $\left\{[g] \in \mathcal{M}(M) \mid \exists f \in[g]\right.$ with $\left.f\left|\alpha=1_{M}\right| \alpha\right\}$.

Theorem 1.1. If $M$ is an atoroidal 3-manifold that contains a genuine lamination, then $\mathcal{M}(M)$ is finite.

Received February 19, 1998. The first author was partially supported by NSF Grant DMS-9505253 and the MSRI. 
Outline. We will assume that $M$ is not Haken since this case was proved by Johannson [8]. It therefore suffices to construct a curve $\alpha \subset M$ such that $I_{\alpha}$ is finite (Lemma 1.2) and such that the set of isotopy classes $\{[f(\alpha)] \mid f \in \operatorname{Homeo}(M)\}$ in $M$ is finite (Lemma 1.4).

For the remainder of the paper we shall assume that $M$ contains a genuine lamination $\lambda$ and is therefore irreducible [7]. We shall also assume that $M$ is not Haken.

Lemma 1.2. If $\alpha \subset M$ is not homotopically trivial, then $I_{\alpha}$ is finite.

Proof. Given an element $g$ of $I_{\alpha}$ we may choose a representative $f$ that preserves a neighborhood $N(\alpha)$ of $\alpha$, setwise. Since $M$ is not Haken, $H_{2}(M)=0$ and it follows that $f: \partial N(\alpha) \rightarrow \partial N(\alpha)$ is isotopic to the identity map. Therefore we may assume that $f \mid N(\alpha)=1_{N(\alpha)}$.

$M-\alpha$ is irreducible since $\alpha$ is homotopically nontrivial. Consider $f \mid(M-\stackrel{N}{N}(\alpha))$. By [8] the quotient of $\mathcal{M}(M-\stackrel{N}{N}(\alpha))$ by the subgroup generated by Dehn twists along annuli and tori is finite. If $A$ is a properly embedded annulus in $M-\stackrel{\circ}{N}(\alpha)$ whose restriction to $\partial N(\alpha)$ is homologically trivial, then a Dehn twist along $A$ is equal, in $\mathcal{M}(M-$ $\stackrel{\circ}{N}(\alpha)$ ), to a Dehn twist along a torus. There is no properly embedded annulus in $M-\stackrel{\circ}{N}(\alpha)$ whose restriction to $\partial N(\alpha)$ is not homologically trivial, for such an annulus would imply the existence of a 1-sided Klein bottle in $M-\stackrel{\circ}{N}(\alpha)$ and since $M$ is not Haken, this would imply that $\pi_{1}(M)$ is finite (it would be 2-fold covered by a lens space), and this contradicts the fact that $\pi_{1}(M)$ is infinite [7].

It follows that up to Dehn twists about tori in $M-\stackrel{\circ}{N}(\alpha)$, there are only finitely many possibilities for $f \mid(M-\stackrel{\circ}{N}(\alpha))$. Since $M$ is atoroidal, Dehn twists along tori are isotopic to $1_{M}$ and hence $I_{\alpha}$ is finite. q.e.d.

We will use the following unique decomposition theorem from [6] to define $\alpha$. This theorem is a natural version of a very easy case of the Jaco-Shalen, Johannson [9], [8] Characteristic Submanifold Theorem.

Lemma 1.3. Let $V$ be the disjoint union of the closed complementary regions of an essential lamination $\lambda$. There exists a unique (up to isotopy in $V$ ) finite collection $\mathcal{A}=A_{1} \cup \cdots \cup A_{n}$ of properly embedded annuli in $V$ such that the following hold:

(1) $V=\mathcal{G} \cup \mathcal{I}$ where $\mathcal{G} \cap \mathcal{I}=\partial_{v} \mathcal{G}=\partial_{v} \mathcal{I}=\mathcal{A}$. 
(2) $\left(\mathcal{I}, \partial_{h} \mathcal{I}\right)$ is an I-bundle over a possibly noncompact or disconnected surface. No component of $\left(\mathcal{I}, \partial_{h} \mathcal{I}\right)$ is an I-bundle over a compact surface with non-empty boundary.

(3) $\left(\mathcal{G}, \partial_{h} \mathcal{G}\right)$ is compact, has no components homeomorphic to $\left(D^{2} \times I, D^{2} \times \partial I\right)$ and contains no essential product disks.

Since $\lambda$ is genuine, the gut, $\mathcal{G}$, is nonempty. Let $Z$ be a component of $\mathcal{A}$ and let $\alpha$ be its core. Recall that $\alpha$ is homotopically non-trivial by $[7]$.

Lemma 1.4. The set of isotopy classes $\{[f(\alpha)] \mid f \in$ Homeo $(M)\}$ in $M$ is finite.

Proof. Let $\Delta$ be a fixed triangulation of $M$. By [3] we may assume that $\lambda$ is maximal gut number lamination and hence, after possibly splitting $\lambda$ along finitely many leaves, $f$ is isotopic to a map, also called $f$, such that $f(\lambda)$ is in normal form with respect to $\Delta$. In the usual way, put an $I$-bundle structure near $f(\lambda)$ so that in each 3 -simplex $\sigma$ of $\Delta$, every maximal collection of parallel normal disks of $f(\lambda) \cap \sigma$ lies in an $I$-fibred $D^{2} \times I$. Thus each $\sigma$ is decomposed into an $I$-bundle region and a non-product region. Let $C$ be the closed complementary region of $\lambda$ containing $\alpha$. Then $f(C)$ can be decomposed as an $I$-bundle, $K$, and a space $H$ which is a finite union of non-product portions of each $\sigma \in \Delta$.

$\partial_{v} H=\partial_{v} K$ is a finite union of annuli. We need to take care of those components which are compressible in $f(C)$. If some component $A$ of $\partial_{v} K$ bounds a $D^{2} \times I \subset V$, with $D^{2} \times \partial I \subset \partial_{h}(f(C))$ and if $\left(\stackrel{\circ}{D}^{2} \times I\right) \cap H \neq$ $\emptyset$, then define $K_{1}=K \cup\left(D^{2} \times I\right)$ and $H_{1}=H-\left(D^{2} \times I\right)$. Note that $K_{1}$ is an $I$-bundle and $H_{1}$ is a union of components of $H$. By continuing in this manner we obtain an $I$-bundle $K_{n}$ such that $H_{n}=\overline{V-K_{n}}$ is a union of components of $H$. Furthermore if $A$ is a component of $\partial_{v} H_{n}$, then either $A$ is $\pi_{1}$-injective in $f(C)$ or $A$ bounds a $D^{2} \times I$ component of $K_{n}$.

It is important to note that no matter which homeomorphism $f$ was initially chosen or how $f(\lambda)$ was put into normal position, by the finiteness of $\Delta$, up to p.l. isotopies of simplices in $\Delta$, there are only 
finitely many $H_{n}$ that can arise in this construction.

\section{FigURE 1}

Let $H^{\prime} \cup K^{\prime}$ be the decomposition of $f(C)$ given by deleting the $D^{2} \times I$ components from $K_{n}$ (if there are any) and adding them to $H_{n}$. The thickened disks that are added to $H_{n}$ to produce $H^{\prime}$ depend on $f$, but since $M$ is irreducible, there is a unique, up to isotopy of $M$, way to add such disks. Thus, up to isotopy of $M$, there are only finitely many $H^{\prime}$ that can arise in this construction.

Since $H^{\prime}$ compact and $\partial_{v} K^{\prime}$ is incompressible, $K^{\prime}$ extends by [9], [8], to a maximal $I$-bundle $L^{\prime} \subset f(C)$. Let $L$ be the $I$-bundle which is a union of those components of $L^{\prime}$ which contain an end of $f(C) . L$ is maximal in the sense that it satisfies (2), (3) of Lemma 1.3. (The $\mathcal{G}$ of (3) is $\overline{f(C)-L}$ and $\partial_{h} \mathcal{G}=\mathcal{G} \cap \partial f(C)$.)

Let $\mathcal{G} \cup \mathcal{I}$ be the decomposition of the closed complementary regions of $\lambda$ given by Lemma 1.3. If $J=\mathcal{I} \cap C$, then $f(J)$ is isotopic to a core of a component of $\partial_{v} L^{\prime}$. Thus, up to isotopy, $f(\alpha)$ is the core of one of finitely many characteristic annuli in the $J S J$ splitting of $H^{\prime}$. Since there are only finitely many possibilities for $H^{\prime}$, Lemma 1.4 follows. q.e.d.

\section{References}

[1] M. Brittenham, Essential laminations and Haken normal form. I, Pacific J. 168 (1995) 217-234.

[2] D. Gabai, Problems in the geometric theory of foliations and laminations on $3-$ manifolds, Geometric Topology (ed. W. H. Kazez), Amer. Math. Soc. Internat. Press 2 (1997) 1-33. 
[3] - Essential laminations and Kneser normal form, Preprint.

[4] D. Gabai \& W.H. Kazez, 3-Manifolds with essential laminations are covered by solid tori, J. London Math. Soc. 47 (1993) 557-576.

[5] - Homotopy, isotopy and genuine laminations of 3-manifolds, Geometric Topology (ed. W.H. Kazez), Amer. Math. Soc. Internat. Press 1 (1997) 123-138.

[6] - Group negative curvature for 3-manifolds with genuine laminations, Geom. Topology 2 (1998) 65-77.

[7] D. Gabai \& U. Oertel, Essential laminations in 3-manifolds, Ann. of Math. (2)130 (1989) 41-73.

[8] K. Johannson, Homotopy equivalences of 3-manifolds with boundary, Lecture Note in Math., Springer, Berlin, Vol. 761, 1983, 1-303.

[9] W. Jaco \& P. Shalen, Seifert Fibered Spaces in 3-Manifolds, Mem. Amer. Math. Soc. 21 (1979).

\section{California Institute of Technology} UNIVERSITY OF GEORGIA 